\title{
Breathing Feedback System with Wearable Textile Sensors
}

\author{
Edmond Mitchell, Shirley Coyle, Noel E O'Connor, Dermot Diamond \\ Centre for Sensor Web Technologies, Dublin City University, CLARITY, Dublin, \\ Ireland \\ Tomas Ward, \\ Biomedical Engineering Group, Dept. of Electronic Engineering, \\ National University of Ireland Maynooth, Ireland
}

\section{ABStraCt}

\begin{abstract}
Breathing exercises form an essential part of the treatment for respiratory illnesses such as cystic fibrosis. Ideally these exercises should be performed on a daily basis. This paper presents an interactive system using a wearable textile sensor to monitor breathing patterns. A graphical user interface provides visual real-time feedback to patients. The aim of the system is to encourage the correct performance of prescribed breathing exercises by monitoring the rate and the depth of breathing. The system is straightforward to use, low-cost and can be installed easily within a clinical setting or in the home. Monitoring the user with a wearable sensor gives real-time feedback to the user as they perform the exercise, allowing them to perform the exercises independently. There is also potential for remote monitoring where the user's overall performance over time can be assessed by a clinician.

keywords:: sensors; signal; wearables; interface; graphical ; user; breathing; system
\end{abstract}

\section{INTRODUCTION}

The average human takes approximately 700,000,000 breaths in their lifetime and each one is critical to maintaining homeostasis. We begin breathing in the womb and despite the briefest of pauses we breathe until literally our "last breath". Breathing is essential for our survival and yet it is generally something people perform without conscious thought. Breathing behaviour can have a profound impact on a person's health[1]. Furthermore, despite the fact that breathing technique can affect our overall health, we receive very little information this in everyday life. For example, by breathing in a slow, steady and deep manner, a person's heartbeat slows and relaxes, blood pressure normalises, stress hormones drop and muscles loosen. These techniques are availed of by athletes to improve performance and reduce stress before and during competitions[2].

There are many diseases that affect the respiratory system in humans. One such disease is asthma, an inflammation of the lungs which causes narrowing of the airways. Asthma affects an estimated 300 million people worldwide. Another is cystic fibrosis, a life-threatening disease which has a high occurrence ratio in Ireland, with one in nineteen people being carriers of the CF gene [3]. While this hereditary disorder affects the entire body, breathing complications are the most serious symptom, and frequent lung infections typically occur.
One of the symptoms of $\mathrm{CF}$ is the accumulation of large amounts of phlegm the lungs. A widely accepted technique to provide relief in such cases is the loosening and removal of phlegm through various breathing practices. The Active Cycle of breathing is one such popular technique that uses breathing exercises to remove phlegm from the lungs. Clearing secretions from peripheral airways is the most important defense mechanism of the respiratory system. The technique involves 4-5 deep breaths coupled with holding periods inbetween to allow air to be transported behind obstructed areas in the lungs. These exercises need to be performed regularly every day[4].

Training the lung muscles can provide significant benefits for patients with respiratory diseases[5]. Regular short shallow breaths can lead to frailties in chest muscle, reduce oxygen circulation, induce shortness of breath and cause poor lung capacity. Proper breathing exercises can help to resolve the severity of these symptoms, increase muscle strength, and improve posture and mental ability [6][7].

Wearable sensor networks have an important role to play in future healthcare delivery and management by sensing the body and interpreting the physiological data[8]. In this work the aim is to develop an interactive system to help patients perform respiratory exercises, and particularly to maintain the interest of children during exercise sessions. This comprises a wearable system which monitors breathing technique and provides visual feedback to the user through a graphical user interface. An additional advantage of this system is exercise sessions can be repeated using the same reference signal, which means that medical staff need not be present for the exercise, thereby improving efficiency in the hospital. Typically these types of exercises require some degree of expert supervision and monitoring. With our system we sought automate at least some part of this process.

\section{System DESCRIPTION}

A system was created to encourage patients to carry out their prescribed breathing exercises. The system was designed for use in both a clinical setting and also for home use where the patient can be remotely monitored. Therefore, it was essential to make the system accessible by ensuring that it was straightforward to install and to operate. The system 


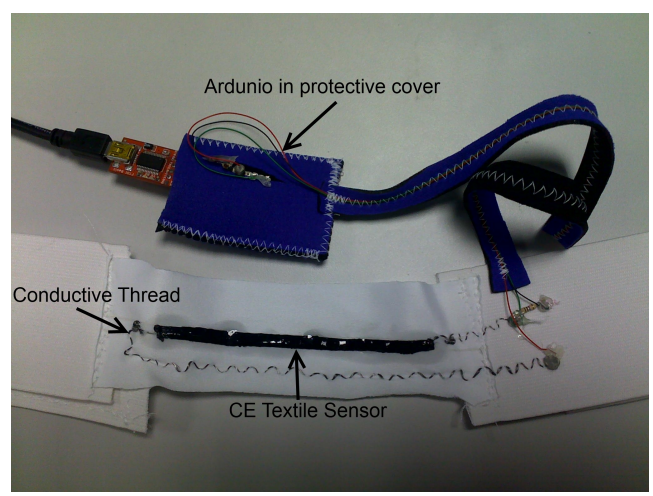

Fig. 1: Respiratory Sensor

also had to be low-cost and compatible with existing computer systems found in hospitals or homes. Sensors embedded in clothing must be comfortable to wear. Therefore, this system consists of a textile sensor which tracks breathing patterns via an embedded microcontroller that is comfortable to wear. The microcontroller was used to sample and transfer the user's breathing signal to a computer for signal processing and analysis. The user interacts with a Graphical User Interface (GUI) which gives real-time feedback of the breathing technique and facilitates continuous assessment of the patient's performance.

\section{A. Hardware}

The textile-based sensor was developed using a piezoresisistive material specifically created to detect body movements [9]. This carbon-loaded elastomer (CE) (WACKER Elastosil 1 LR 3162 A/B) had previously been used to measure body kinematics by the researchers at the University of Pisa[6]. As the material is stretched, its resistance increases. This effect can be used to detect joint movement and also to measure breathing rates. Breathing rate may be measured by monitoring the expansion and contraction of the ribcage. Placement of the sensors at the chest and abdomen allows patterns of breathing to be monitored, e.g. shallow breathing versus deep abdominal breathing. CE textile sensors are made by masking a region on the fabric and then coating it with the elastomer substance, followed by curing for $2 \mathrm{hrs}$ at $80 \mathrm{C}$. The CE sensitive strip was coated over an embroidered knot of conductive thread (Bekinox 100\% stainless steel 2-ply yarn), and connected in series with a resistor to create a voltage divider circuit. A source voltage of $3.3 \mathrm{~V}$ was applied to the series resistors and a voltage was measured across the $\mathrm{CE}$ sensor to detect its response. The $\mathrm{CE}$ sensor was coated with silicone to protect it from moisture and environmental effects. This helps to increase the lifetime of the sensor and maximise robustness during wear. The sensor was integrated into an elastic chest strap as shown in Figure 1. The size of the strap is adjustable by means of a Velcro attachment.

A shirt with a number of sensors has been developed to measure the expansion of the thoracic/abdominal cavity, as shown in Figure 2. The shirt is made from a stretch knit

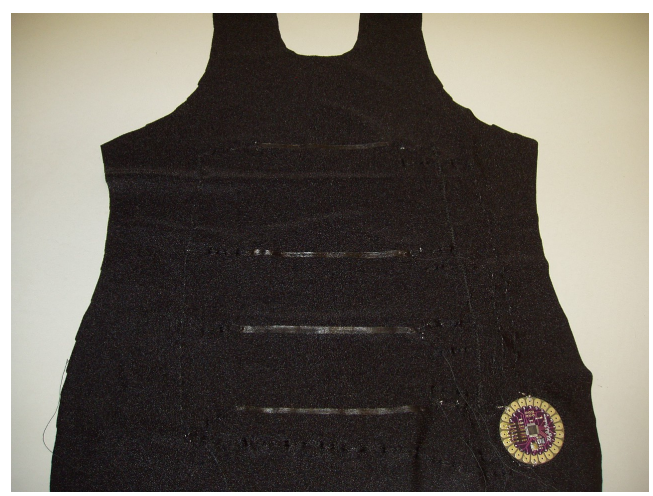

Fig. 2: Breathing monitoring t-shirt

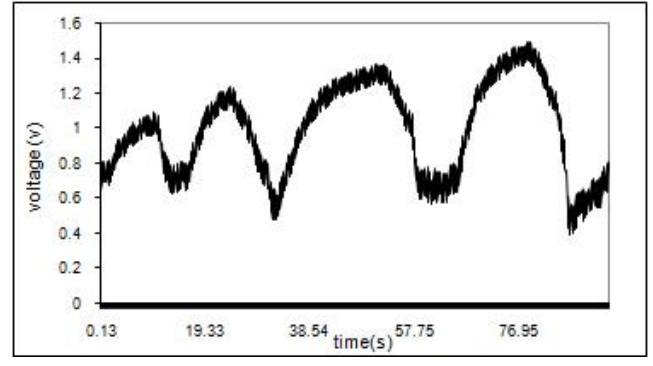

Fig. 3: Unfiltered Breathing Signal

fabric ( $80 \%$ nylon, $20 \%$ lycra). In order to maximise the signal quality, the sensors need to be coupled closely with the body movements, and therefore the garment needs to fit the wearer well. For greater flexibility the chest strap was developed to allow a universal fit during clinical trials.

The Digi Xbee wireless radio frequency module was used to sample data from the sensor. This allowed data to be transferred to a PC while granting unobtrusive monitoring of the lung muscle. It has an indoor/urban range of up to 90 metres. The receiver connects to the computer through USB and a virtual COM port (VCP) drivers. It has 10-bit resolution which is an adequate range for resolving the small differences in measurement. It a theoretical maximum sampling rate of $65 \mathrm{kHz}$ and contains six analog to digital convertor channels (ADCs). The use of the Xbee extended the capability of the system compared to the previous wired solution. These areas include its longer range and higher data capture rates. For example it allows the monitoring of athletes on the football pitch and the running track. Real-time feedback for coaches enables training tactics to be adapted to maximise training output [10]. Physiological data of each athlete can be logged over training sessions to analyse their response to training and monitor their fitness. A typical breathing signal measured using the textile sensor system is shown in Figure 3.

\section{B. Signal Analysis}

The software provides instruction and real-time feedback to the user and also records the data so that the user's performance can be logged over time. The software allows 
the patient and doctor/therapist to perform a breathing exercise for a specified length of time which the patient must attempt to emulate. This signal is recorded as a reference breathing signal.An example of this can be seen in Figure 4 As the exercise is being performed, the breathing signal is graphed in real time in the bottom right corner of the screen. Once the reference signal has been recorded it is then used to instruct the user for future exercises. This dynamic method of instructing correct breathing technique caters to various breathing patterns. Instruction is given to the user by means of an avatar, whose mouth expands and contracts in time with the breathing sequence. As the user watches the avatar performing they must attempt to emulate it. The user's breathing signal is compared in real-time to the reference signal and immediate feedback is given to the user. An overlay image of a mouth represents the user's breathing as seen in Figure 10. Therefore as they perform the exercise they must try to follow the breathing sequence of the avatar and synchronise the two mouths.

Each signal is filtered with a 3rd order digital low-pass Butterworth filter with a cut off at $1 \mathrm{~Hz}$. This filter reduces noise from the breathing sensor. The raw data signal as shown in Figure 3 has been filtered and is displayed in Figure 4. Filtering the data ensures a smooth transition of the avatar's feedback allowing the user to emulate it more easily. When

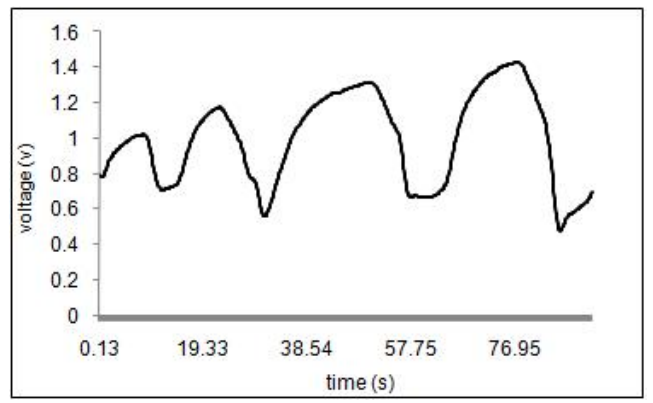

Fig. 4: Filtered Breathing Signal

the patient has completed the exercise, they are presented with a number of stars depending on their performance. In order to calculate the number of stars a user receives, a measurement of how similar the signals were would need to be determined. One such way to ascertain this was to calculate the correlation coefficient. Equation (1) was used to correlate the reference signal and the emulated signal.

$$
R=\frac{\sum x_{i} y_{i}-\frac{\sum x_{i} \sum y_{i}}{N}}{\sqrt{\left(\sum x_{i}^{2}-\frac{\left(\sum x_{i}\right)^{2}}{N}\right)\left(\sum y_{i}^{2}-\frac{\left(\sum y_{i}\right)^{2}}{N}\right)}}(1)
$$

Where $R$ is the normalised cross correlation coefficient, $x$ is the reference signal, $y$ is the emulated signal and $N$ is the number of samples. $R$ values are most sensitive to similarities and discrepancies in shape. It is also very sensitive to timing and can be used to find correlations between signals with inherent delay. Figure 6 shows three different breathing signals recorded by the system. The solid (light blue) line is the reference signal which consisted of seven slow deep breaths

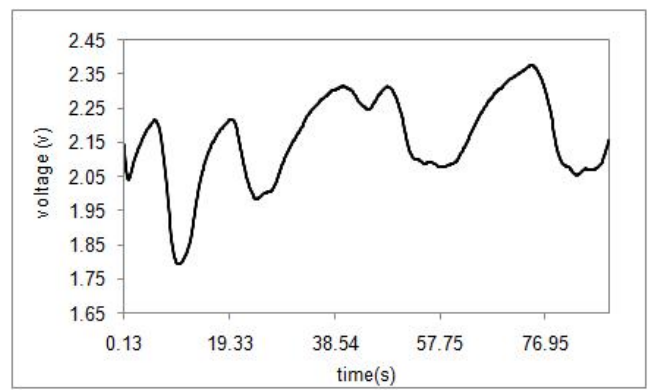

Fig. 5: Emulated Filtered Signal

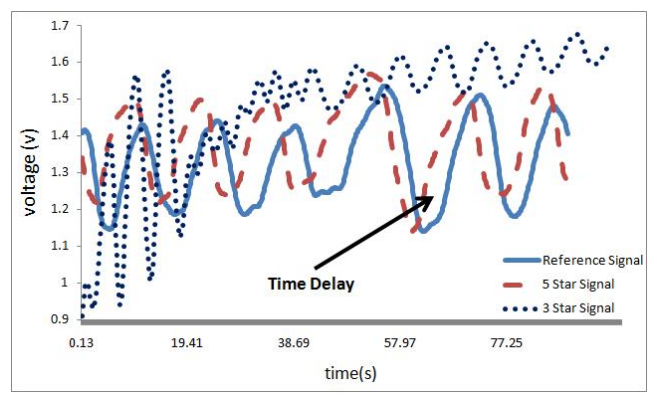

Fig. 6: Comparisons of different signal Grades

over the course of 90 seconds. Two emulated signals were compared to it. The dashed (red) line represents a trial where the user emulated the avatars breathing very accurately. This performance was given a five star grading. The dotted (dark blue) line represents a trial where the user performed short shallow breaths that did not synchronise well with the avatar's breathing. The amplitude change of this signal is smaller than the five star signal as the sensor is not stretched as much during shallow breathing. This performance was given a three star grading. The grading system was designed to be supportive so that it would be encouraging and provide positive feedback to the user for carrying out the exercise in the first instance.

From Figure 6 it can be seen that there is a slight delay between the avatar's instruction and the user's performance of the task. Therefore the program first needed to calculate this time delay before grading the user. The correlation function was calculated for the reference and emulated signals. The system implements a sliding window to identify the time lag between the signals by finding the index of the maximum correlation coefficient. For the reference signal and five star trial, the maximum correlation coefficient was found to be 0.9242 at a time lag of 24 samples $(3.08 \mathrm{sec})$. Figure 7 shows

TABLE I: Sample Correlation Values

\begin{tabular}{|l|c|}
\hline $\begin{array}{l}\text { Signal cross correlated with } \\
\text { reference signal }\end{array}$ & $\mathrm{R}$ \\
\hline 5 Star & 0.2839 \\
3 Star & 0.1686 \\
5 Star with optimum time shift & $0.9242 @+24$ samples \\
3 Star with optimum time shift & 0.3020 @ - 17 samples \\
\hline
\end{tabular}




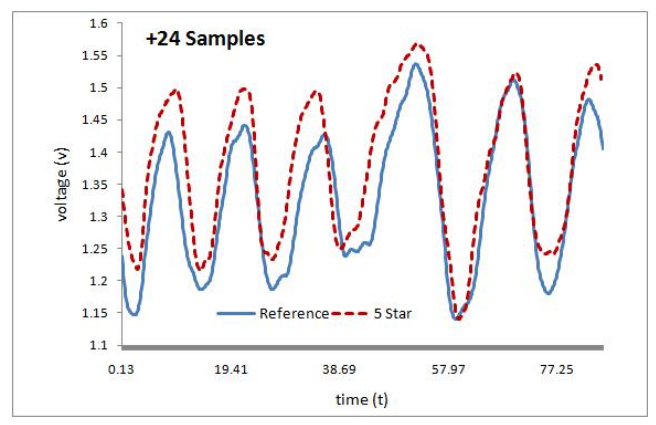

Fig. 7: Shifting the signal to find optimum correlation

these signals with the time shift applied.

Various tests were conducted in order to ascertain the grading of user signals based on the normalised cross correlation coefficient $\mathrm{R}$. Three grades were created based on star-grading, with 5 stars corresponding to full marks and 3 stars corresponding to the poorest grade. From Figure 6 and Figure 7 it can be seen that the 5 star signal closely resembles the reference signal. With this data and similar data from other tests it was empirically decided that users who received over 0.85 for $R$ deserved 5 stars. The other grades were calculated in the same way. From Figure 6 it can be seen that there is little resemblance between the 3 star signal and the reference signal. Even with signal time shift the maximum cross correlation coefficient was low. Any user that receives a coefficient of less than 0.7 receives 3 stars while if they receive a score between 0.7 and 0.85 a 4 star grade is awarded.

Three stars were assigned to the poorest grade in order to encourage the user to keep continuing with the breathing training. The primary target population for this feedback system is children, and it is extremely important to keep them well motivated and focused on improving their technique.

\section{Graphical Interface}

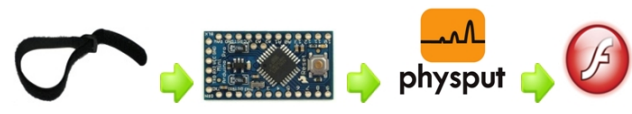

Fig. 8: Flowchart of data passing through system

The software was developed using $\mathrm{C \#}$ and Adobe Flash. $\mathrm{C \#}$ gathers the data from the serial port and converts it into key presses, as flash has a limited number of inputs, one of which is key presses. The software downloads the latest flash file from the internet, and opens it in a window at maximum resolution.

Physput (physiological input) is software which facilitates the easy acquisition of data from sensor devices, allowing incoming data to be easily converted to GUI events. These events include mouse clicks, keyboard presses and mouse movements [11].

The Flash application allows the user to record breathing signals, and replay them subsequently at their leisure. Flash was used because it can create graphically rich applications quickly that are extremely user friendly. Children respond well to graphically rich applications and bright colours and flash lent itself to creating a friendly-faced avatar [12]. It has an extensive user base with $99.3 \%$ of internet desktop users having it installed, which allows easy distribution of the software. The software checks for an updated version of the Flash file upon start up and automatically downloads the newer version. This requires no action from the user thus making their interaction with the system much simpler.

One important feature that was added to the application was a real-time filtered plot of the reference signal. This can be seen in the bottom right corner of the screen as shown in Figure 9. As the patient records the reference signal the therapist can both view the graph and the patient. This allows therapist to ensure the patient is performing the breathing exercise properly. In Figure 10 a patient is attempting to

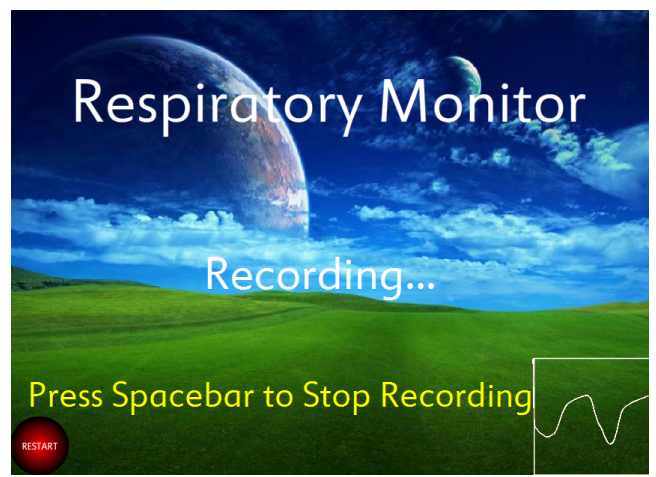

Fig. 9: Reference Signal Recording with Real-time graphing.

emulate the reference signal. This screen contains a grey mouth that expands and contracts in unison with the recorded reference signal. It also has another mouth, in blue, which expands and contracts in synchronisation with the patients' current breathing signal. This visual feedback allows the patient to replicate the reference signal more easily. An interactive system to help patients perform respiratory exercises, and particularly to sustain children's attention during these exercise sessions was developed. This system records the signals from

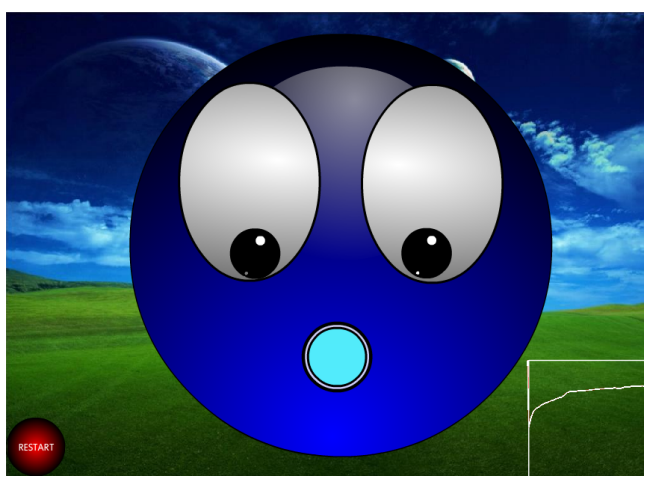

Fig. 10: Graphical User Interface - User attempting to emulate reference signal 


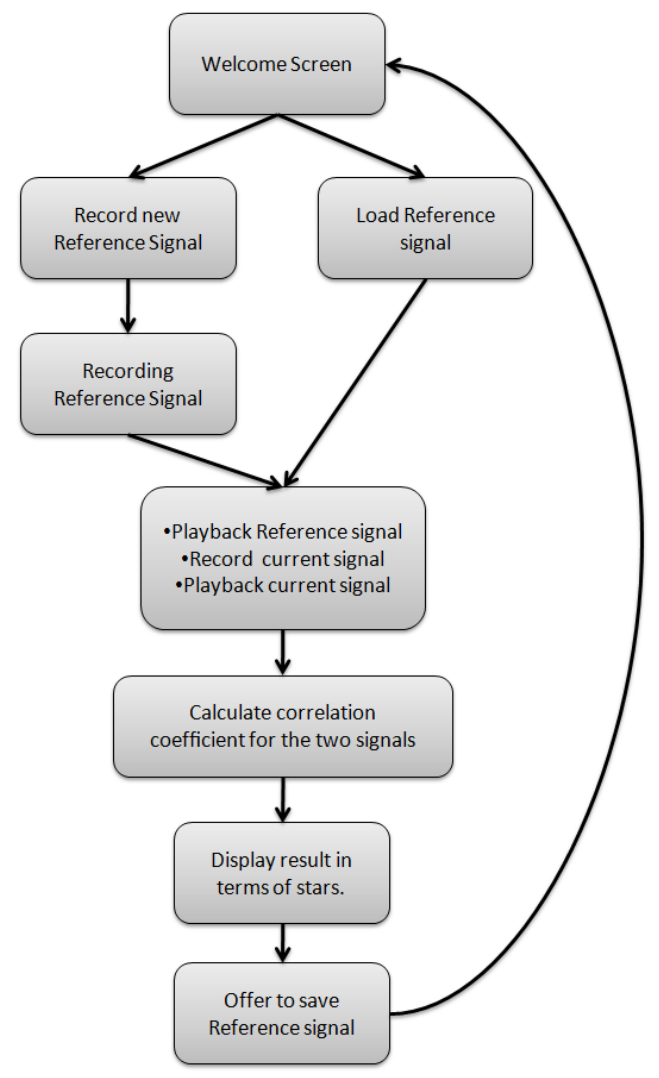

Fig. 11: State Machine Diagram

a polymer sensor which is fully integrated into a garment, and provides immediate feedback to the user. It can be used at home, while also facilitating remote supervision by a trained therapist who can provide regular assessment updates. The use of Flash makes the software easily upgradeable. It also allows automatic logging of results to a personalised webpage which is remotely accessible by the therapist.

\section{CONCLUSIONS}

The advantage of our system is that it is low-cost and the sensor garment is flexible and comfortable to wear. This low-cost sensor could be mass-produced by screen-printing processes commonly used in the textile industry. The use of Flash makes the software easily accessible and visually stimulating. Visual interaction is extremely important in order to keep users interested and alert. The software is very easy to use, as it comprises of only three buttons once the Flash application has been launched. Future work will focus on an assessment of sensor robustness and the importance of garment fit for clinical trials. Discussions with healthcare specialists are in progress to obtain early feedback on system usability in a clinical setting

\section{FUTURE WORK}

The visualisation of data is imperative in improving the overall user experience. This, combined with encouraging

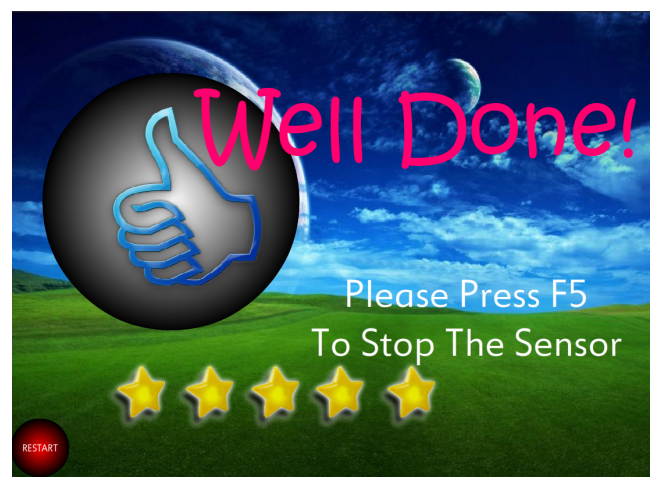

Fig. 12: Result Screen

feedback, helps retain users and grow the user base. It is also important to work with potential users of the system from the start of the design process. This allows the system to be custom-built to suit their needs and takes a multi-disciplinary approach to system development.

Future work will focus on the possible amalgamation of sensors into a single garment. Seamless integration into clothing would allow non-invasive monitoring of physiological signals. With the use of wireless transceivers, it may be possible to integrate sensors into everyday garments and connect the sensor output to a watch-like or mobile phone platform. In this way the task can become part of the wearer's daily routine. Different levels in a gaming application could be developed with user specific backgrounds or avatars. More specific training games could be designed to cater for sufferers of different diseases such as chronic obstructive pulmonary disease (COPD) or for the aged. Further work on investigating breathing patterns could prove fruitful to see whether breathing patterns can give some early indication of respiratory disease or injury. There are clearly many potential implementations of the system in healthcare and in other application domains in which breathing technique is important such as sport, yoga and theatrical musical performance.

A clinical trial is being planned in a Dublin Hospital to investigate the viability of this training regimen. This trial will investigate the benefits of the system as a training tool for CF patients to help improve lung function. Non CF patients' breathing data will be used as a comparison. This will allow patients to give feedback on the operation of the current system as well as evaluating how motivating the system is. 


\section{ACKNOWLEDGEMENTS}

This work is supported by Science Foundation Ireland under grant 07/CE/I1147. We would like to thank the staff at the The William Stokes Institute, Adelaide and Meath Hospital, Tallaght, Dublin 24, Ireland.

\section{REFERENCES}

[1] Leon Chaitow, Dinah Bradley, and Chris Gilbert. Multidisciplinary Approaches to Breathing Pattern Disorders. Churchill Livingstone, 1 edition, 2002.

[2] L Garza. Implementing a breathing technique to manage performance anxiety in softball. The Sport Journal, 12(2), 2009.

[3] Cystic fibrosis association of ireland -. http://www.cfireland.ie/, January 2010.

[4] A Hristara-Papadopoulou and J Tsanakas. Results of active cycle of breathing techniques and conventional physiotherapy in mucociliary clearance in children with cystic fibrosis. Hippokratia, 11(4):202-204.

[5] C Kigin. Breathing exercises for the medical patient: The art and the science. Physical Therapy, 70(11):700-706, November 1990.

[6] F Carpi and D De Rossi. Electroactive polymer-based devices for etextiles in biomedicine. IEEE Transactions on Information Technology in Biomedicine, 9(3):295-318.

[7] S Enright and K Chatham. Inspiratory muscle training improves lung function and exercise capacity in adults with cystic fibrosis* CHEST CHEST, 126(2):405-411, August 2004.

[8] D Diamond, S Coyle, S Scarmagnani, and J Hayes. Wireless sensor networks and Chemo-/Biosensing. Chemical Reviews, 108((2)):652679, 2008.

[9] S Coyle, D Morris, K.T Lau, N Moyna, and D Diamond. Textile-based wearable sensors for assisting sports performance.

[10] Noel E O'Connor, P. Kelly, C. O'Conaire, D. Connaghan, Alan Smeaton, B. Caulfield, D. Diamond, and N. Moyna. Tennissense: A multi-modal sensing platform for sport. EICIM News, Special Edition on the Sensor Web, 2009.

[11] E Mitchell. Physput - software for mapping physiological signals to standard computer input. http://www.eeng.nuim.ie/ tward/software/physput.html, January 2010.

[12] Julie A. Jacko and Andrew Sears. The Human-Computer Interaction Handbook: Fundamentals, Evolving Technologies and Emerging Applications. Lawrence Erlbaum, 1 edition, September 2002. 